\title{
Intoxicação por Solanum fastigiatum (Solanaceae) em bovinos: epidemiologia, sinais clínicos e morfometria das lesões cerebelares $^{1}$
}

\author{
Raquel R. Rech², Daniel R. Rissi², Aline Rodrigues², Felipe Pierezan ${ }^{3}$, José V.M. \\ Piazer $^{4}$, Glaucia D. Kommers ${ }^{5}$ e Claudio S.L. Barros ${ }^{5 *}$
}

\begin{abstract}
Rech R.R., Rissi D.R., Rodrigues A., Pierezan F., Piazer J.V.M., Kommers G.D. \& Barros C.S.L. 2006. [Poisoning by Solanum fastigiatum (Solanaceae) in cattle: epidemiology, clinical signs and morphometry of cerebellar lesions.] Intoxicação por Solanum fastigiatum (Solanaceae) em bovinos: epidemiologia, sinais clínicos e morfometria das lesões cerebelares. Pesquisa Veterinária Brasileira 26(3):183-189. Departamento de Patologia, Universidade Federal de Santa Maria, 97105900 Santa Maria, RS, Brazil. E-mail: claudioslbarros@uol.com.br

Nineteen cases of Solanum fastigiatum (Solanaceae) poisoning in cattle from three municipalites in the State of Rio Grande do Sul, Brazil, occurring from 2003 to 2005, are described. Morbidity and mortality rates were respectively $6.7 \%$ and $3.4 \%$. Average age of affected cattle was five-yearold (2 to 8-year-old) and duration of clinical courses was 3-18 months. Clinical signs observed in all affected cattle were cerebellar deficits characterized by hypermetry, incoordination, falls, muscle tremors, transitory seizures and wide base stance. One affected bovine had encephalic traumatic subdural hemorrhage and another had gross atrophy of the cerebellum. Histologically, lesions were restricted to the cerebellum and consisted of partial or complete vacuolation of the perikaria of Purkinje neurons with occasional axonal spheroids in the granular cell layer and in the white matter of the cerebellum. In advanced cases there were extensive loss of cerebellar Purkinje neurons and proliferation of the Bergmann's glia. The morphometric evaluation of the numbers of Purkinje neurons and of the thickness of the cerebellar molecular layer indicated decreased numbers of Purkinje neurons with consequent decrease in the molecular layer thickness.
\end{abstract}

INDEX TERMS: Poisonous plants, Solanum fastigiatum, Solanaceae, lysosomal storage diseases, central nervous system, diseases of cattle, neuropathology.

RESUMO.- Dezenove casos de intoxicação por Solanum fastigiatum são descritos em bovinos de três municípios do Rio Grande do Sul no período de 2003-2005. Os coeficientes de morbidade e mortalidade foram respectivamente de $6,7 \%$ e $3,4 \%$. Os bovinos afetados tinham idade média de 5 anos (2-8 anos) e a evolução clínica variou de 3-18 meses. Os sinais clínicos obser-

\footnotetext{
${ }^{1}$ Recebido em 17 de fevereiro de 2006.

Aceito para publicação em 23 de fevereiro de 2006.

Parte da Tese de Doutorado do primeiro autor.

${ }^{2}$ Programa de Pós-graduação em Medicina Veterinária (PPGMV), Universidade Federal de Santa Maria (UFSM), área de concentração em Patologia Veterinária.

${ }^{3}$ Bolsista de Iniciação Científica do CNPq junto ao Departamento de Patologia da UFSM.

${ }^{4}$ Inspetoria Veterinária de Jaguari, RS, Rua Carlos Calegaro 290, Jaguari, RS 97760-000.

${ }^{5}$ Depto Patologia, UFSM, Santa Maria, RS 97105-900, Brasil. *Autor para correspondência: claudioslbarros@uol.com.br.
}

vados em todos os bovinos foram de disfunções cerebelares caracterizadas por hipermetria, incoordenação, quedas, tremores musculares, convulsões esporádicas e permanência em estação com os membros em base ampla. Em um bovino foi observada hemorragia traumática subdural no encéfalo e em outro, atrofia macroscópica do cerebelo. As lesões histológicas eram confinadas ao cerebelo e consistiram de vacuolização parcial ou total do pericário dos neurônios de Purkinje com ocasionais esferóides axonais na camada de células granulares e na substância branca cerebelar. Nos casos avançados, havia acentuada perda dos neurônios de Pukinje e proliferação dos astrócitos de Bergmann. $O$ estudo morfométrico do número de neurônios de Purkinje e da espessura da camada molecular revelou um decréscimo no número de células de Purkinje que se correlacionou com conseqüiente diminuição da espessura da camada molecular.

TERMOS DE INDEXAÇÃO: Plantas tóxicas, Solanum fastigiatum, Solanaceae, intoxicação por planta, doença de depósito lisossomal, sistema nervoso central, doenças de bovinos, neuropatologia. 


\section{INTRODUÇÃO}

Doenças de depósito lisossomal (DDL) caracterizam-se pelo acúmulo de substratos não-metabolizados nos lisossomos, resultante da atividade deficiente de hidrolases ácidas. Em animais, ocorrem dois tipos de DDL: as hereditárias, devidas a diferentes mutações genéticas, e as adquiridas, causadas pela ingestão de plantas tóxicas (Jolly \& Walkley 1997, Summers et al. 1995).

Em bovinos, as DDL de origem genética são pouco comuns e incluem glicogenose tipo II em Brahman e Shorthorn, gangliosidose $\mathrm{GM}_{1}$ em Holandês, $\alpha$-manosidose em Aberdeen Angus, Murray Grey e Galloway, $\beta$-manosidose em bovinos Salers e Simental e ceróide-lipofuscinose em Beefmaster e Devon (Jolly \& Walkley 1997, Van der Lugt 2002). A documentação da ocorrência de glicogenose hereditária em bovinos Brahman (Zlotowski et al. 2005) constitui o único relato de DDL de origem genética em bovinos no Brasil. DDL adquiridas em ruminantes incluem $\alpha$-manosidoses secundárias à ingestão de plantas do gênero Swainsona na Austrália e Oxytropis e Astragalus na América do Norte, América do Sul, China eÁfrica (Van der Lugt 2002). No Brasil, DDL induzidas pela ingestão de plantas incluem manosidoses causadas por Ipomoea carnea subsp. fistulosa (Riet-Correa et al. 2003) e Sida carpinifolia (Driemeier et al. 2000), lipofuscinose causada por Phalaris angusta (Barros et al. 2006) e uma neurolipidose causada por Solanum fastigiatum (RietCorrea et al. 1983b, Barros et al.1987, Paulovich et al. 2002).

S. fastigiatum ocorre no sul do Brasil e Uruguai como um arbusto que atinge até $1 \mathrm{~m}$ de altura, com folhas largas e flores brancas. É invasora de pastagens ou de terrenos abandonados e conhecida popularmente como "joá-preto" ou “jurubeba”. Para que ocorra a intoxicação é necessário que os bovinos consumam grandes quantidades da planta; a doença caracteriza-se por sinais neurológicos causados por vacuolização dos neurônios de Purkinje (Riet-Correa et al. 1983b, Barros et al. 1987). A ingestão de Solanum $k$ webense, $S$. dimidiatum e $S$. bonariensis induz em bovinos, respectivamente, da África do Sul (Pienaar et al. 1976), dos Estados Unidos (Menzies et al. 1979) e do Uruguai (Riet-Correa et al. 1983b), sinais clínicos e achados anatomopatológicos semelhantes à intoxicação por S. fastigiatum. S. viarum e S. cinereum causam intoxicação semelhante em caprinos, respectivamente, na Austrália e nos Estados Unidos (Bourke 1997, Porter et al. 2003).

Entre 2003 e 2005, durante o diagnóstico de rotina foram examinados 301 encéfalos de bovinos enviados ao Laboratório de Patologia Veterinária (LPV) da Universidade Federal de Santa Maria (UFSM), como parte do sistema de vigilância da encefalopatia espongiforme bovina (BSE), coordenada pelo Programa Nacional de Controle da Raiva dos Herbívoros e Outras Encefalopatias (PNCRH) do Ministério da Agricultura Pecuária e Abastecimento (MAPA) do Brasil. Desses, 19 eram casos de intoxicação por $S$. fastigiatum. Os dados epidemiológicos, sinais clínicos, achados anatomopatológicos e o estudo histomorfométrico das lesões cerebelares desses casos são descritos.

\section{MATERIAL E MÉTODOS}

O material deste estudo consistiu de 301 encéfalos de bovinos examinados no LPV/UFSM; desses, 221 apresentavam sinais clínicos de distúrbios nervosos. Históricos, sinais clínicos e achados de necropsia de bovinos com doença neurológica foram obtidos por visita às propriedades e dos arquivos da Inspetoria Veterinária dos municípios de Jaguari, Santiago e Itaara, no Rio Grande do Sul. Os encéfalos foram fixados em formol a $10 \%$ e os seguintes fragmentos foram selecionados para exame histológico: (1) bulbo na altura do óbex, (2) cerebelo, (3) ponte com pedúnculos cerebelares, (4) mesencéfalo na altura dos colículos rostrais (5) seção do diencéfalo através da massa intermédia e (6) córtex frontal na altura do joelho do corpo caloso e dos núcleos da base. Esses fragmentos foram processados rotineiramente para histologia e corados pela hematoxilina e eosina (Barros \& Marques 2003).

Para o estudo morfométrico do cerebelo, foi selecionada uma fatia longitudinal do verme cerebelar dos 19 bovinos intoxicados por Solanum fastigiatum e de 9 bovinos sem sinais clínicos de distúrbios nervosos e lesões no encéfalo, que formaram o grupo controle (Grupo C). Os neurônios de Purkinje foram contados em 20 campos microscópicos com objetiva 10. Em cada campo eram contados todos os neurônios de Purkinje de uma folha cerebelar. Nos cerebelos com lesões histológicas de intoxicação por $S$. fastigiatum, quando a média do número de neurônios de Purkinje foi inferior a 10, os casos foram agrupados no Grupo A; aqueles em que a contagem da média de neurônios foi superior a 10 foram alocados no Grupo B. Para avaliar a espessura da camada molecular, foram realizadas 3 medidas/folha (faces laterais e ápice da folha) nas mesmas folhas cerebelares em que foram contados os neurônios de Purkinje, utilizando ocular micrométrica ${ }^{6}$.

\section{RESULTADOS}

A intoxicação por Solanum fastigiatum foi diagnosticada em 6,3\% dos 301 encéfalos de bovinos examinados, e em $8,6 \%$ de bovinos com sinais clínicos de distúrbios nervosos. Os dados epidemiológicos das propriedades estão listados no Quadro 1. Os bovinos tinham idade média de 5 anos (2-8 anos) e pastejavam em

Quadro 1. Históricos das propriedades rurais onde ocorreram casos de bovinos intoxicados por Solanum fastigiatum

\begin{tabular}{cccccccc}
\hline $\begin{array}{c}\text { Bovino Número de } \\
\text { no. }\end{array}$ & proxo & Idade & Raça & $\begin{array}{c}\text { Propri- } \\
\text { edade }\end{array}$ & $\begin{array}{c}\text { Município } \\
\text { (RS) }\end{array}$ & $\begin{array}{c}\text { Número de } \\
\text { bovinos na } \\
\text { propriedade }\end{array}$ \\
\hline S1 & DX 0263/03 & Fa $^{\mathrm{a}}$ & 8 anos & SRD $^{\text {b }}$ & A & Jaguari & 23 \\
S2 & DX 0273/03 & MC $^{c}$ & 8 anos & SRD & B & Jaguari & 09 \\
S3 & DX 0281/03 & F & 4 anos & SRD & C & Jaguari & 11 \\
S4 & DX 0325/03 & F & 7 anos & SRD & D & Jaguari & 19 \\
S5 & DX 0063/04 & MC & 3 anos & SRD & E & Jaguari & 33 \\
S6 & DX 0073/04 & MC & 4 anos & SRD & F & Jaguari & 113 \\
S7 & DX 0078/04 & F & 4 anos & Nelore & G & Jaguari & 91 \\
S8 & DX 0155/04 & MC & 5 anos & Holandês & H & Jaguari & 21 \\
S9 & DX 0164/04 & F & 4 anos & Holandês & E & Jaguari & - \\
S10 & DX 0256/04 & F & 4 anos & Charolês & E & Jaguari & - \\
S11 & DX 0019/05 & F & 6 anos & SRD & G & Jaguari & - \\
S12 & DX 0037/05 & F & 6 anos & SRD & G & Jaguari & - \\
S13 & DX 0038/05 & F & 4 anos & SRD & G & Jaguari & - \\
S14 & DX 0084/05 & Me & 2 anos & Holandês & I & Jaguari & 11 \\
S15 & DX 0163/05 & F & 4 anos & SRD & G & Jaguari & - \\
S16 & DX 0242/05 & F & n.i. & Holandês & J & Santiago & 139 \\
S17 & DX 0305/05 & F & 7 anos & SRD & L & Jaguari & 40 \\
S18 & DX 0353/05 & F & 2 anos & SRD & M & Itaara & 21 \\
S19 & DX 0423/05 & MC & 4 anos & Holandês & N & Jaguari & 21
\end{tabular}

aFêmea, bsem raça definida, c macho castrado, d morte espontânea, ${ }^{\mathrm{e}}$ macho,

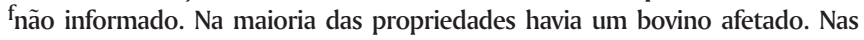
propriedades $\mathrm{A}, \mathrm{E}, \mathrm{G}, \mathrm{H}, \mathrm{M}$ havia respectivamente, 2, 6, 16, 2 e 3 bovinos afetados.

\footnotetext{
6 Ocular micrométrico de tornillo para mediciones exactas bajo el microscópio. E. Leitz/Wetzlar, Alemanha.
} 

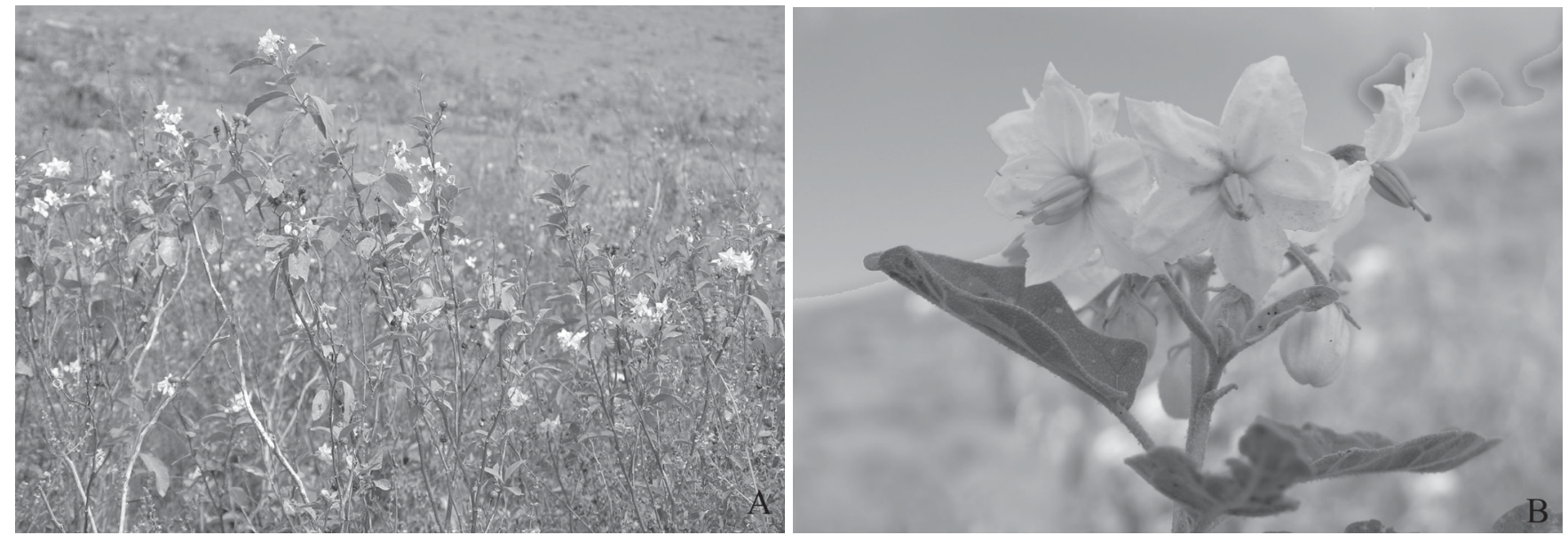

Fig.1. A. Campo com grande quantidade de exemplares de Solanum fastigiatum. B. Detalhe da planta em floração.

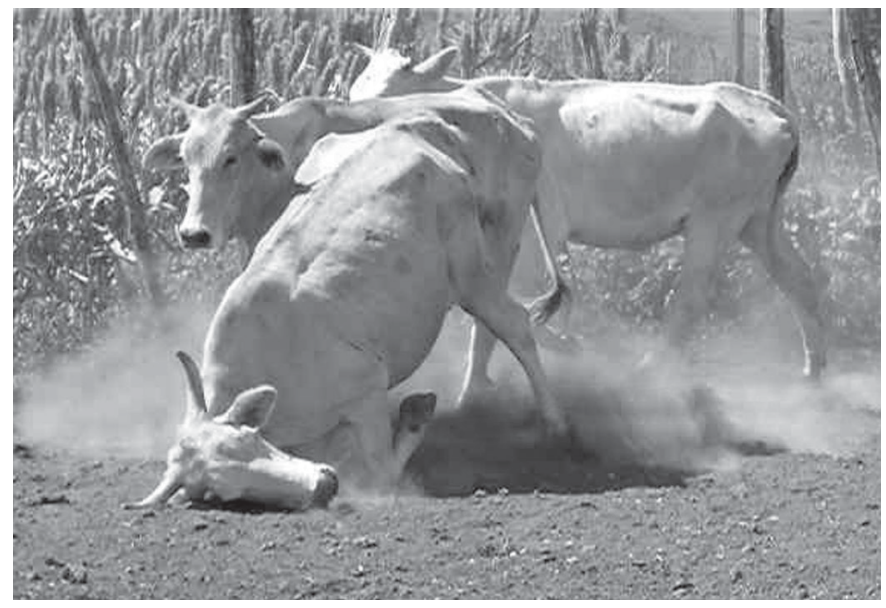

Fig.2. Bovino em queda, com crises convulsivas provocadas pela intoxicação por Solanum fastigiatum.

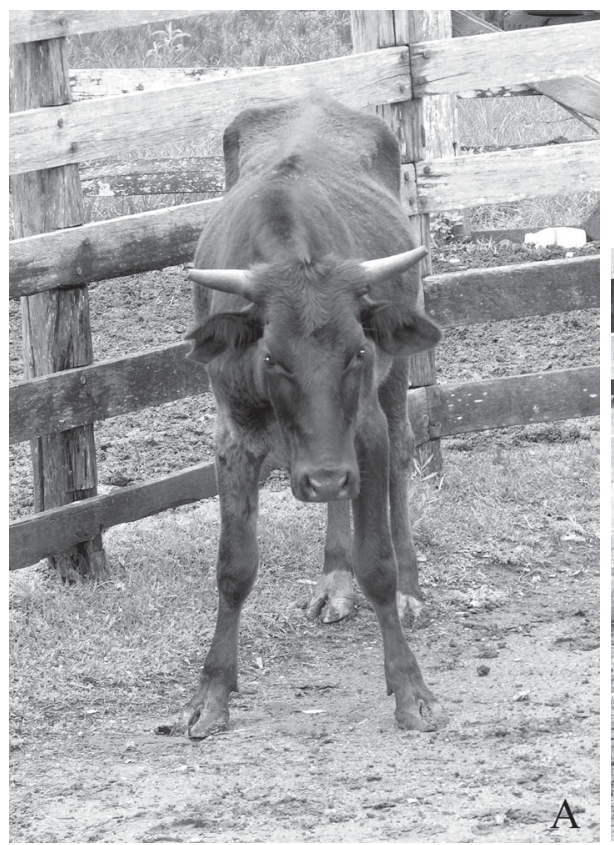

campos infestados por grande quantidade de S. fastigiatum (Fig.1). Os índices de morbidade e mortalidade foram, respectivamente de, 6,7\% e 3,4\%. Em seis casos (S1, S2, S6, S9, S18 e S19) o tempo de evolução do quadro clínico foi informado e variou de 3 meses a 1 ano e meio. Clinicamente, além de emagrecimento progressivo, todos os bovinos apresentavam sinais de distúrbios nervosos caracterizados por graus variáveis de hipermetria, incoordenação, quedas e tremores musculares quando movimentados (Fig.2), excitados, ou presos por cordas. Apresentavam convulsões esporádicas ou frequientes que duravam alguns segundos e eram seguidas de recuperação. Em alguns bovinos, para verificar os sinais nervosos, foi aplicado o teste conhecido como head raising test, que consiste em levantar a cabeça do animal durante um minuto e soltá-la logo após; se houver convulsão, o teste é positivo (Pienaar et al. 1976). Após as crises, alguns bovinos permaneciam com os membros em posição de base ampla, para tentar manter o equilíbrio (Fig.3). Escoriações ocorriam na pele como resultado de traumatismo. Um dos bovinos morreu afogado em um açude e os demais foram eutana-

Fig.3. A. Bovino com os membros torácicos entreabertos, em posição de base larga, na tentativa de manter o equilíbrio. B. Bovino com os membros pélvicos estendidos para trás.

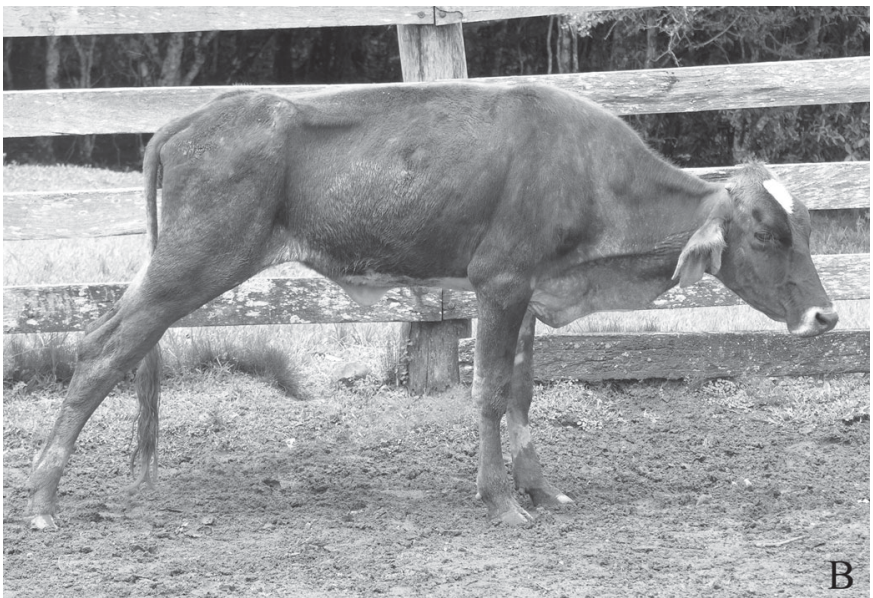




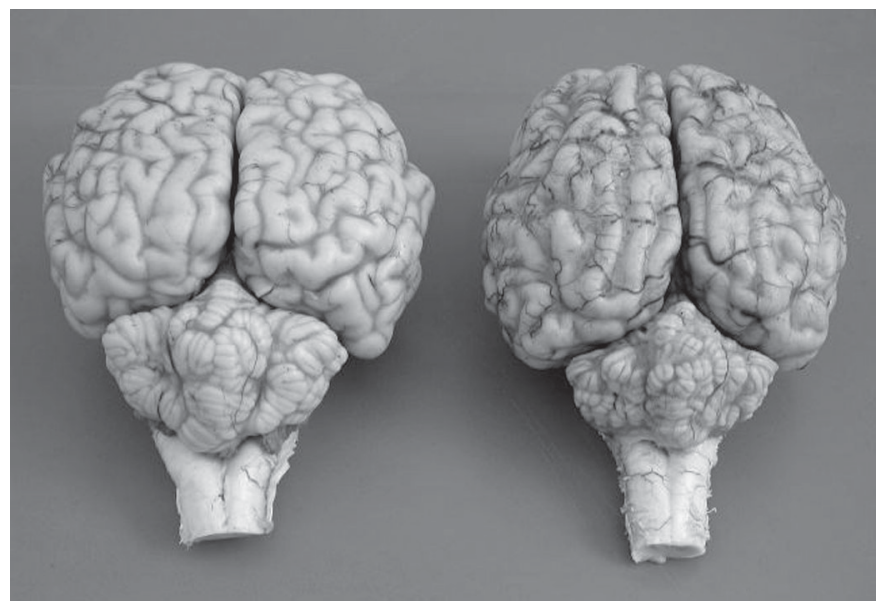

Fig.4. Atrofia do cerebelo (à direita) em bovino intoxicado por Solanum fastigiatum. À esquerda, encéfalo normal de bovino para comparação.

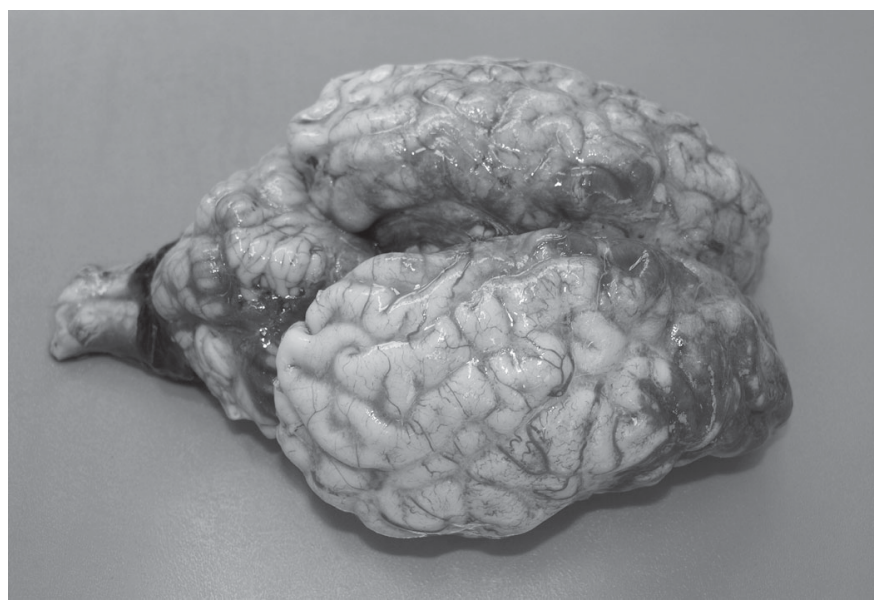

Fig.5. Hemorragia subdural na superfície dorsal do telencéfalo e do cerebelo decorrentes de repetidas quedas. siados. Em uma visita à Propriedade G, o proprietário relatou que cerca de 3 bovinos intoxicados pela planta, morreram durante a queda de um morro íngreme (em razão das convulsões) e fraturaram a coluna vertebral. Nessa mesma propriedade havia cerca de 300 caprinos que ingeriam a planta, mas não apresentavam sinais clínicos de distúrbios nervosos. Achados macroscópicos foram observados em dois bovinos. Em um deles havia atrofia moderada do cerebelo (Fig.4), e em outro, hemorragia subdural na superfície dorsal do telencéfalo e do cerebelo (Fig.5), provavelmente causada por traumatismos provenientes de quedas.

As lesões histológicas observadas em todos os casos consistiam de fina vacuolização parcial ou total do pericário dos neurônios de Purkinje (Fig.6a), que por vezes, localizava-se na periferia do pericário. Em alguns casos, a vacuolização era tão acentuada que o núcleo dos neurônios de Purkinje era deslocado para a periferia, e em outros observavam-se poucos e grandes vacúolos intracitoplasmáticos. Havia degeneração dessas células, com perda da substância de Nissl e eosinofilia da região citoplasmática imediatamente ao redor do núcleo (Fig.6b). Em casos mais avançados, havia perda acentuada dos neurônios de Purkinje, com ocasional rarefação dessa camada e proliferação dos astrócitos de Bergmann ao redor dos espaços deixados pelos neurônios (Fig.7). Quantidades variáveis de esferóides axonais com 10-40 $\mu \mathrm{m}$ de diâmetro, eram observadas na camada de célu-

Quadro 2. Resultados da média, desvio padrão e coeficiente de variância do número de neurônios de Purkinje e da espessura da camada molecular no cerebelo dos bovinos dos Grupos A, B e C

\begin{tabular}{lccccccc}
\hline Parâmetros & \multicolumn{2}{c}{$\begin{array}{c}\text { Número de neurônios } \\
\text { de Purkinje }\end{array}$} & & \multicolumn{2}{c}{$\begin{array}{c}\text { Espessura da camada } \\
\text { molecular }\end{array}$} \\
\cline { 2 - 4 } \cline { 6 - 8 } & Grupo A Grupo B & \multicolumn{2}{c}{ Grupo C } & & Grupo A Grupo B Grupo C \\
\hline Média & 3,8 & 18,0 & 16,9 & & 214,3 & 327,4 & 345,1 \\
Desvio padrão & 2,4 & 2,2 & 2,1 & & 34,0 & 29,7 & 15,8 \\
$\begin{array}{l}\text { Coeficiente de } \\
\text { variância (\%) }\end{array}$ & 61,4 & 12,5 & 12,5 & & 15,9 & 9,1 & 4,6 \\
& & & & & & &
\end{tabular}
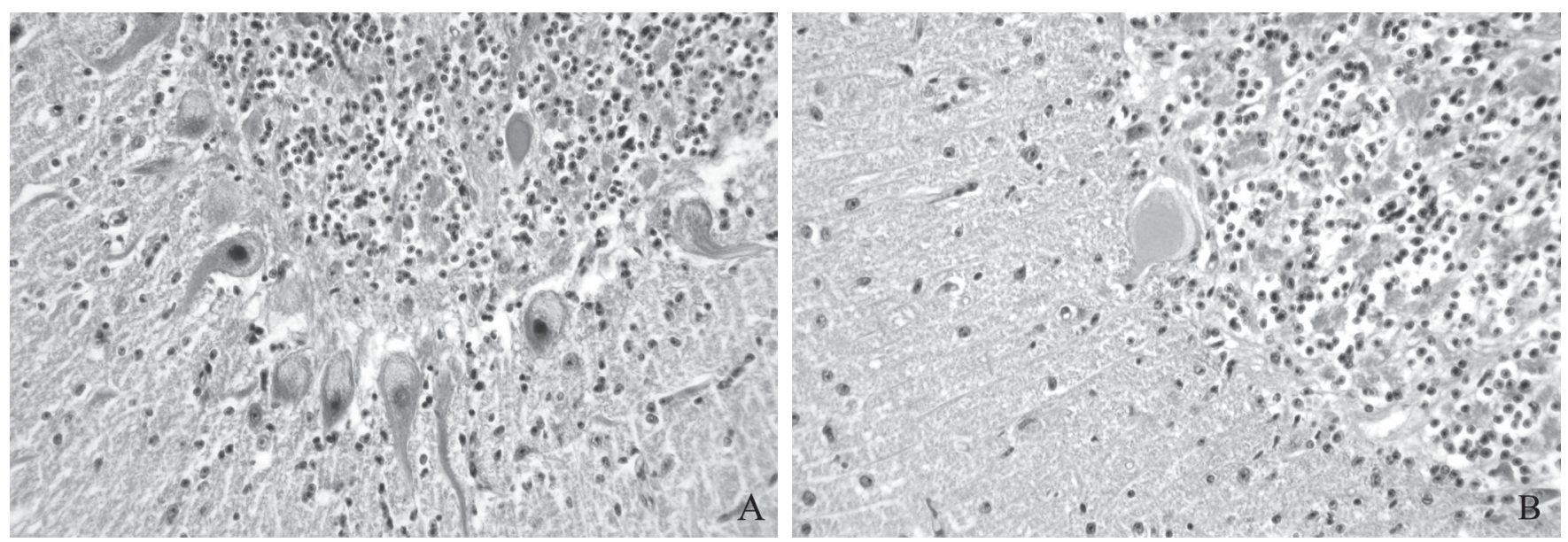

Fig.6. A. Fina vacuolização do pericário dos neurônios de Purkinje e esferóide axonal na camada de células granulares em um bovino intoxicado por Solanum fastigiatum (Grupo B). HE, obj.10. B. Degeneração de um neurônio de Purkinje com fina vacuolização citoplasmática periférica (Grupo B). HE, obj.40. 


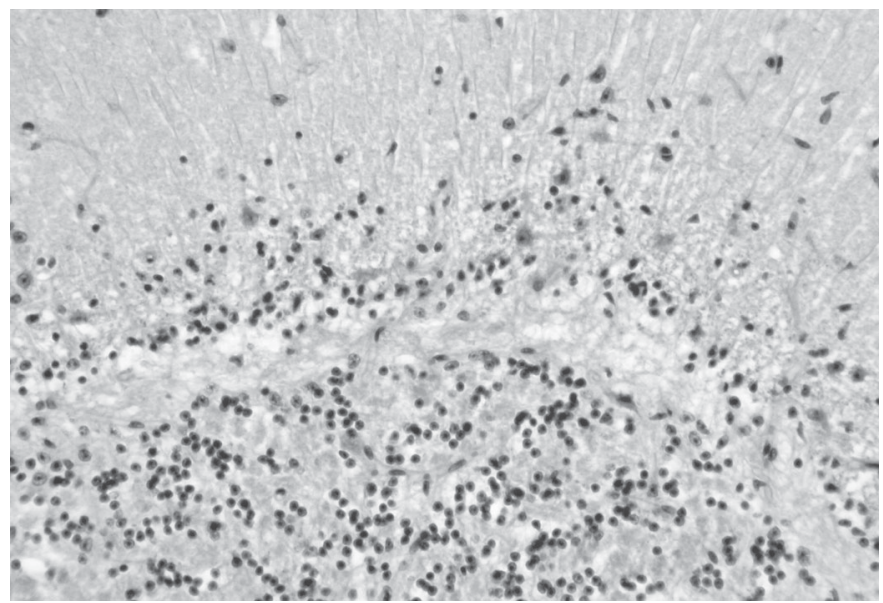

Fig.7. Perda total dos neurônios de Purkinje e substituição por astrócitos de Bergmann em um caso avançado de intoxicação por Solanum fastigiatum (Grupo A). HE, obj.20
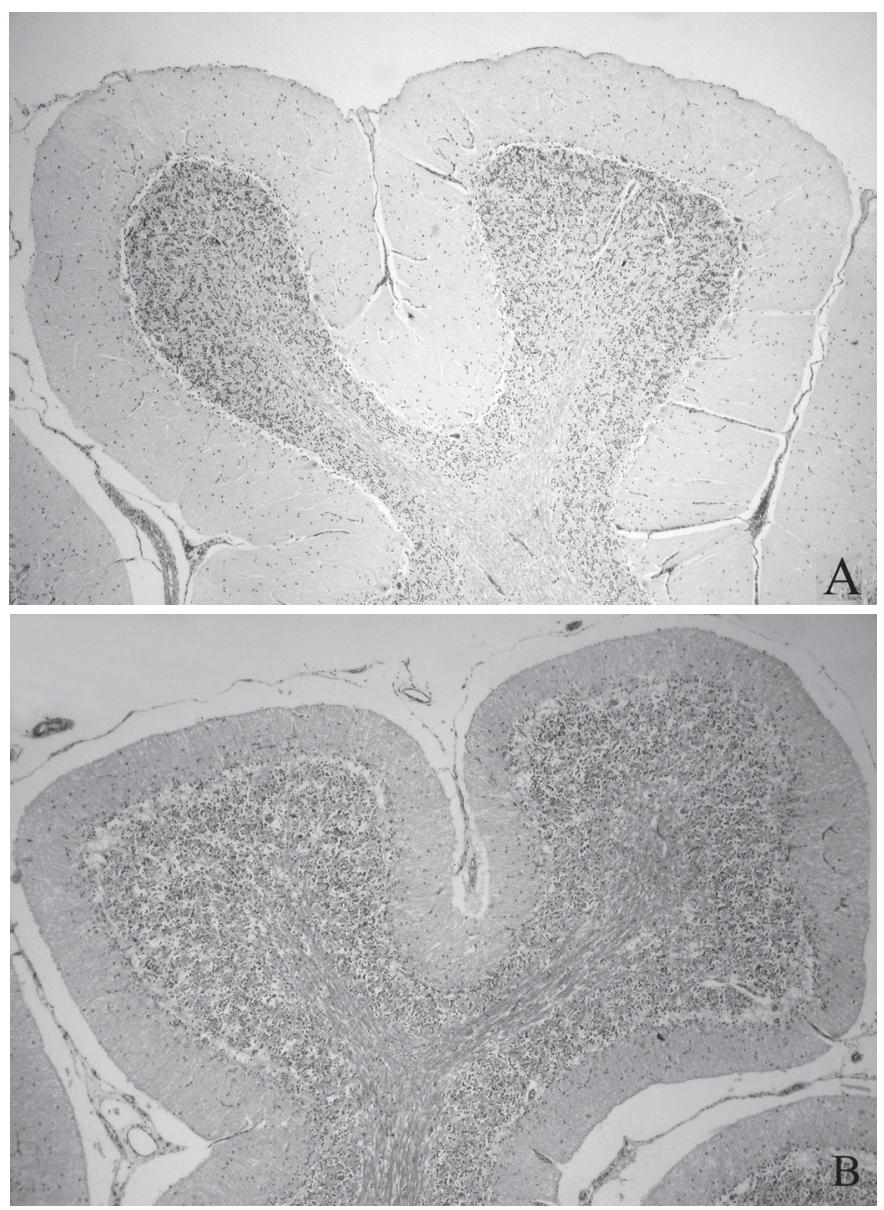

Fig.8. A. Cerebelo de bovino normal (Grupo C). HE, obj.10. B. Cerebelo de bovino intoxicado por Solanum fastigiatum (Grupo A). Observe o adelgaçamento da camada molecular. HE, obj.10.

las granulares e na substância branca do cerebelo. Focos de gliose também foram observados na substância branca do cerebelo. O número médio de neurônios de Purkinje e da espessura da camada molecular nos Grupos A, B e C estão listados no

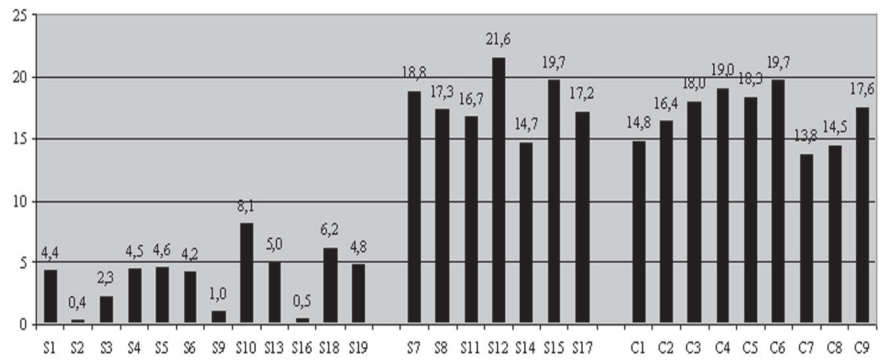

Fig.9. Representação gráfica da média do número de neurônios de Purkinje de cada folha cerebelar nos três grupos examinados. Observa-se a acentuada diminuição do número de neurônios nos bovinos do Grupo A (cerebelos de bovinos intoxicados por Solanum fastigiatum com número médio inferior a 10 neurônios de Purkinje/ folha) quando comparada ao Grupo B (cerebelos de bovinos intoxicados por $S$. fastigiatum com número médio superior a 10 neurônios de Purkinje/folha) e ao Grupo C (cerebelos controle).

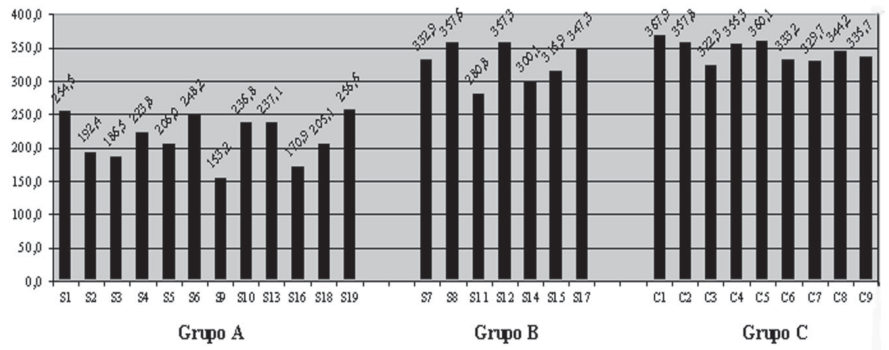

Fig.10. Representação gráfica da análise morfométrica da camada molecular do cerebelo nos três grupos examinados. Observa-se acentuada diminuição da espessura da camada molecular nos bovinos do Grupo A (cerebelos de bovinos intoxicados por Solanum fastigiatum com número médio inferior a 10 neurônios de Purkinje/ folha) e leve diminuição na espessura da camada molecular nos bovinos do Grupo B (cerebelos de bovinos intoxicados por $S$. fastigiatum com número médio superior a 10 neurônios de Purkinje/ folha) em relação àqueles do Grupo $\mathrm{C}$ (cerebelos controle).

Quadro 2. Demonstrou-se uma redução da camada molecular do Grupo A de 34,5\% em relação ao Grupo B e de 37,9\% em relação Grupo C (Fig.8). 0 estudo morfométrico dos neurônios de Purkinje e da camada molecular revelou alta correlação $(0,92)$ entre o número de neurônios e a espessura desta camada nos cerebelos (Fig. 9 e 10).

Outros achados histológicos no encéfalo incluíram: autólise da camada de células granulares do cerebelo, discretos manguitos perivasculares, vacuolização citoplasmática dos neurônios do núcleo vermelho e lipofuscinose neuronal; esses são considerados achados incidentais por vários autores (Summers et al. 1995, Gavier-Widen et al. 2001).

\section{DISCUSSÃO}

O diagnóstico de intoxicação por Solanum fastigiatum foi baseado nos históricos, sinais clínicos e achados histológicos. A intoxicação produz doença neurológica em bovinos caracterizada por déficits cerebelares e tem índices de morbidade (1-20\%) e mortalidade baixos (Riet-Correa et al. 1983b, Barros et al. 1988), como observado nas propriedades aqui estudadas. 
Sinais clínicos de distúrbios cerebelares são descritos em bovinos com doenças congênitas e hereditárias como infecções virais intra-uterinas causadas pelo vírus da diarréia viral bovina, abiotrofias cerebelares, ataxia cerebelar em Aberdeen Angus e Charolês, hipoplasia e degeneração cerebelares em bovinos Hereford, Shorthorn, Ayrshire e Angus e em doenças de depósito lisossomal, principalmente as manosidoses (Mayhew 1989). No Brasil, dentre esses grupos de doenças congênitas já foram relatadas: degeneração cerebelar cortical em bovinos Holandês, hipermetria hereditária em bovinos Shorthorn, hipomielinogênese congênita, hipoplasia cerebelar em bovinos Charolês (Schild 2001) e glicogenose generalizada em Brahmans (Zlotowski et al. 2005). Geralmente, em todas essas doenças, os sinais clínicos aparecem logo após o nascimento ou nas primeiras semanas ou meses de idade (Scarratt 2004).

Apesar da intoxicação por S. fastigiatum produzir sinais clínicos característicos de disfunção cerebelar, ela deve ser incluída no diagnóstico diferencial de outras doenças do sistema nervoso central em ruminantes adultos que cursam com sinais clínicos semelhantes. No Brasil, destacam-se as doenças tremorgênicas causadas pela intoxicação pelo fungo Claviceps paspali, que parasita gramíneas do gênero Paspalum na região sul (Riet-Correa et al. 1983a) e pela intoxicação por Ipomoea asarofolia que ocorre nas regiões Norte e Nordeste (Döbereiner et al. 1960, RietCorrea et al. 2003). Nessas intoxicações não são observadas lesões histológicas, e a recuperação dos animais afetados ocorre logo após a retirada dos animais dos campos infestados (Tokarnia et al. 2000, Barros et al. 2006).

Vacuolização neuronal de aspecto finamente granular não é um achado específico da intoxicação por $S$. fastigiatum e ocorre em outras doenças de herbívoros causadas pela ingestão de plantas do gênero Solanum (Pienaar et al. 1976, Menzies et al. 1979, Bourke 1997, Porter et al. 2003), Swainsona, Oxytropis, Astragalus (Summers et al. 1995), Ipomoea (Van der Lugt 2002) e Sida (Driemeier et al. 2000, Loretti et al. 2003b, Furlan 2005, Seitz et al. 2005); e nas DDL hereditárias (Jolly \& Walkley 1997). Intoxicação por Prosopis juliflora e pelo fungo Aspergillus clavatus não são DDL, mas induzem vacuolização neuronal em neurônios do tronco encefálico de bovinos. P. juliflora causa a doença denominada "cara torta" no semi-árido nordestino (Riet-Correa et al. 2003) e a intoxicação por A. clavatus ocorre quando bovinos são alimentados com resíduos de cervejaria contaminados pelo fungo (Loretti et al. 2003a).

No Brasil, plantas que causam vacuolização neuronal cerebelar em ruminantes domésticos incluem Ipomoea fistulosa, syn. I. carnea (Armien 2000), e I. riedelli (Riet-Correa et al. 2003); mas a epidemiologia dessas duas intoxicações difere grandemente da intoxicação por S. fastigiatum; I. carnea e I. riedelli ocorrem no Nordeste e afetam principalmente ovinos e caprinos (Tokarnia et al. 2000). Estudos de microscopia eletrônica revelaram que os vacúolos dos neurônios de Purkinje na intoxicação por S. fastigiatum correspondem a inclusões membranosas lipídicas semelhantes às encontradas nas neurolipidoses de animais e humanos (Barros et al. 1987). Isso parece ter sido confirmado por estudos histoquímicos com lectinas, que sugerem que a intoxicação é uma doença de depósito glicolipídico (Paulovich et al. 2002).
Atrofia cerebelar ocorre nas abiotrofias cerebelares descritas em caninos, felinos, bovinos, ovinos e eqüinos e se caracteriza pela degeneração do córtex cerebelar, com perda das células de Purkinje após o nascimento (Summers et al. 1995, Fox et al. 2000); e nas abiotrofias múltiplas sistêmicas descritas em cães, eqüinos e felinos (Fox et al. 2000, Résibois \& Poncelet 2004), onde há degeneração cerebelar e de outros locais funcionalmente não relacionados ao cerebelo. Além dessas condições de aparecimento precoce, atrofia do córtex cerebelar é relatada na intoxicação por S. kwebense (Van der Lugt et al. 2002) e I. fistulosa (Armien 2000), como descrito nos casos aqui examinados. Apesar de ser descrito que a falta de neurônios de Purkinje, provoca a falsa impressão de que a camada molecular está adelgaçada (Bourke 1997), através do estudo morfométrico dos casos aqui examinados, demonstrou-se que a redução da espessura da camada molecular é um achado real: entre o Grupo C (controle) e o grupo severamente afetado (Grupo A) a redução foi de $37,9 \%$. Além disso, o coeficiente de correlação entre o número de neurônios de Purkinje e a espessura da camada molecular foi alto. Os valores da espessura da camada molecular entre o Grupo $\mathrm{B}$ e o Grupo $\mathrm{C}$, apresentaram pequena diferença; isso pode ser explicado pelo fato de que no Grupo B, apesar de vacuolizados, os neurônios de Purkinje estavam em números semelhantes aos do Grupo C (controle). Já no Grupo A, havia acentuada diminuição do número de neurônios de Purkinje, e conseqüentemente, nesses casos, o número de dendritos na camada molecular tem de ser acentuadamente menor, levando conseqüientemente à diminuição da espessura dessa camada. A recuperação dos bovinos afetados ocorre quando os bovinos são retirados das áreas com $S$. fastigiatum, logo após o aparecimento dos sinais clínicos. No entanto, neste estudo, todos os animais que apresentaram curso clínico longo pertenciam ao Grupo A. Esses animais foram submetidos a eutanásia quando não havia indicação que viessem a se recuperar da doença. $O$ estudo morfométrico das lesões cerebelares desses casos permite concluir que bovinos com lesões avançadas acabam morrendo por acidentes que sofrem durante as crises epileptiformes ou são eutanasiados em decorrência da má condição corporal. A recuperação desses animais torna-se inviável devido à irreversiblidade das lesões cerebelares.

\section{REFERÊNCIAS}

Armién A.G. 2000. Vergleichende klinische und morphologische Untersuchungen zur spontanen und experimentellen Vergiftung durch Ipomoea fistulosa (Convolvulaceae) bei Ziegen. Dissertation, Institut für Veterinär-Pathologie der Justus-Liebig-Universität Gießen, Alemanha. 211p.

Barros C.S.L. \& Marques G.H.F. 2003. Procedimentos para o diagnóstico das doenças do sistema nervoso central de bovinos. Ministério da Agricultura, Pecuária e Abastecimento, Departamento de Defesa Animal, Brasília. 50p. Disponível na internet: http://www.agricultura.gov.br

Barros C.S.L., Jardim Filho J.O., Alves C.T.M. \& Conrado L.F.I. 1988. Intoxicação em bovinos por Solanum fastigiatum var. fastigiatum. $10^{\circ}$ Congr. Estadual Med. Vet., Porto Alegre, p.87. (Resumo)

Barros C.S.L., Driemeier D., Dutra I.S. \& Lemos R.A.A. 2006. Doenças do Sistema Nervoso de Bovinos no Brasil: Coleção Vallée. AGNS, São Paulo. 207p.

Barros S.S., Riet-Correa F., Andujar M.B., Barros C.S.L., Méndez M.C. \& Schild A.L. 1987. Solanum fastigiatum and Solanum sp poisoning in cattle: ultraestructural changes in the cerebellum. Pesq. Vet. Bras. 7:1-5. 
Bourke C.A. 1997. Cerebellar degeneration in goats grazing Solanum cinereum (Narrawa burr). Aust. Vet. J. 5:363-365.

Döbereiner J., Tokarnia C.H. \& Canella C.F.C. 1960. Intoxicação experimental pela "salsa" (Ipomoea asarifolia R. et Schult ) em ruminantes. Arqs Inst. Biol. Animal, Rio de J., 3:39-57.

Driemeier D., Colodel E.M., Gimeno E.J. \& Barros S.S. 2000. Lysosomal storage disease caused by Sida carpinifolia poisoning in goats. Vet. Pathol. 37:153-159.

Fox J., Duncan R., Friday P., Klein B. \& Scarrrat W. 2000. Cerebello-olivary and lateral (accessory) cuneate degeneration in a juvenile American miniature horse. Vet. Pathol. 37:271-274.

Furlan F.H., Lucioli J., Medeiros A. \& Gava A. 2005. Sida carpinifolia em bovinos - relato de caso. $12^{\circ}$ Enapave, Belo Horizonte, p.9. (Resumo)

Gavier-Widen D., Wells G.A.H., Simmons M.M., Wilesmith J.W. \& Ryan J. 2001. Histological observations on the brains of symptomless 7-yearold cattle. J. Comp. Path. 124:52-59.

Jolly R.D. \& Walkley S.U. 1997. Lysosomal storage diseases of animals: an essay in comparative pathology. Vet. Pathol. 34:527-548.

Loretti A.P., Colodel E.M., Driemeier D., Corrêa A.M., Bangel Jr J.J. \& Ferreiro L. 2003a. Neurological disorder in dairy cattle associated with consumption of beer residues contaminated with Aspergillus clavatus. J. Vet. Diagn. Invest. 15:123-132.

Loretti A.P., Colodel E.M., Gimeno E.J. \& Driemeier D. 2003b. Lysosomal storage disease in Sida carpinifolia toxicosis: an induced mannosidosis in horses. Eq. Vet. J. 35:434-438.

Mayhew I.G. 1989. Large Animal Neurology: a handbook for veterinary clinicians, p.227-242. Lea \& Febiger, Philadelphia. 380p.

Menzies J.S., Bridges C.H. \& Bayley Jr E.M. 1979. A neurological disease of cattle associated with Solanum dimidiatum. Southwest. Vet. 32:45-49.

Paulovich F.B., Portiansky E.L., Gimeno E.J., Schild A.L., Mendez M.C. \& Riet-Correa F. 2002. Lectin histochemical study of lipopigments present in the cerebellum of Solanum fastigiatum var. fastigiatum intoxicated cattle. J. Vet. Med. A. Physiol. Pathol. Clin. Med. 49:473-477.

Pienaar J.G., Kellerman T.S., Basson P.A., Jenkins W.L. \& Vahrmeijer J. 1976. Maldronksiekte in cattle: a neuronopathy caused by Solanum kwebense N.E. Br. Onderstepoort J. Vet. Res. 43:67-74.

Porter M.B., MacKay R.J., Uhl E., Platt S.R. \& de Lahunta A. 2003. Neurologic disease putatively associated with ingestion of Solanum viarum in goats. J. Am. Vet. Med. Assoc. 223:501-504.

Résibois A. \& Poncelet L. 2004. Olivopontocerebellar atrophy in two adults cats, sporadic cases or new genetic entity. Vet Pathol. 41:20-29.

Riet-Correa F., Schild A.L., Méndez M.C., Tavares A.S. \& Rodriguez J.O. 1983a. Intoxicação por Claviceps paspali em bovinos no Rio Grande do Sul. Pesq. Vet. Bras. 3:59-65.

Riet-Correa F., Méndez M.C., Schild A.L., Summers B.A. \& Oliveira J.A. 1983b. Intoxication by Solanum fastigiatum var. fastigiatum as a cause of cerebellar degeneration of cattle. Cornell Vet. 73:240-256.

Riet-Correa F., Tabosa I.M., Azevedo E.O., Medeiros R.M.T., Simões S.V.D., Dantas A.F.M., Alves C.J., Nobre V.M.T., Athayde A.C.R., Gomes A.A. \& Lima E.F. 2003. Semi-árido em Foco, Patos-UCG, 1(1):56-57.

Scarratt W.K. 2004. Cerebellar disease and disease characterized by dysmetria or tremors. Vet. Clin. North Am. Food Anim Pract. 20:275-286.

Schild A.L. 2001. Defeitos congênitos, p.19-43. In: Riet-Correa F., Schild A.L., Méndez M.C. \& Lemos R.A.A. (ed.) Doenças de Ruminantes e Eqüinos. $2^{\mathrm{a}}$ ed. Vol.1. Varella, São Paulo. 425p.

Seitz A.L., Colodel E.M., Schmitz M., Gimeno E.J. \& Driemeier D. 2005. Use of lectin histochemistry to diagnose Sida carpinifolia (Malvaceae) poisoning in sheep. Vet. Rec. 156:386-388.

Summers B.A., Cummings J.F. \& de Lahunta A. 1995. Veterinary Neuropathology. Mosby, St Louis. 527p.

Tokarnia C.H., Döbereiner J. \& Peixoto P.V. 2000. Plantas Tóxicas do Brasil, p.120-144. Editora Helianthus, Rio de Janeiro. 310p.

Van der Lugt J.J. 2002. Lysosomal storage diseases, p.15-16. In: Van der Lugt J.J. (ed.) The Clinicopathology and Pathology of Selective Toxicoses and Storage Diseases of the Nervous System of Ruminants in Southern Africa. Dissertation, University of Utrecht, The Netherlands. 174p.

Van der Lugt J.J., Bastianello S.S., Van Ederen A.M. \& Van Wilpe E. 2002. Cerebellar cortical degeneration in cattle caused by Solanum kwebense, p.49-66. In: Van der Lugt J.J. (ed.) The Clinicopathology and Pathology of Selective Toxicoses and Storage Diseases of the Nervous System of Ruminants in Southern Africa. Dissertation, University of Utrecht. 174p.

Zlotowski P., Nakazato L., Dutra V., Barros S.S., Gimeno E.J., Göcks M., Colodel E.M. \& Driemeier D. 2005. Glicogenose hereditária em bovinos Brahman no Brasil. Pesq. Vet. Bras. 25:210-214. 